\title{
Overexpression of a stilbene synthase gene from Fallopia multiflora causes trans-piceid accumulation in transgenic Arabidopsis
}

\author{
S. SHENG ${ }^{1}$, W. XIA ${ }^{2}$, W. RUI ${ }^{3}$, W. ZHAO ${ }^{2}$, T. SHE ${ }^{1}$, J. XIE ${ }^{1}$, S. ZHAO ${ }^{2 *}$, and L. CHEN ${ }^{4 *}$ \\ College of Biology and Food Engineering, Guangdong University of Education, \\ Guangzhou 510225, P.R. China ${ }^{1}$ \\ School of Food Science and Engineering, South China University of Technology, \\ Guangzhou 510641, P.R. China ${ }^{2}$ \\ Center for Drug Research and Development, Guangdong Pharmaceutical University, \\ Guangzhou 501006, P.R. China \\ Shenzhen Research Institute, State Key Laboratory of Agrobiotechnology, The Chinese University of Hong \\ Kong, Shenzhen 518057, P.R. China ${ }^{4}$
}

\begin{abstract}
Fallopia multiflora, also known as Polygonum multiflorum, is a traditional Chinese medicine with stilbenes as the main characteristic components. We previously isolated the presumed stilbene synthase gene FmSTS1 from F. multiflora and inserted the FmSTS1 gene into the genome of Arabidopsis thaliana under the control of cauliflower mosaic virus $35 \mathrm{~S}$ promoter to evaluate its biological functions. Southern blot and Northern blot analyses were performed to confirm the integration and expression of FmSTS1 in Arabidopsis. A new stilbene compound, which was identified as trans-piceid, was found to accumulate in the transgenic Arabidopsis. This study firstly reportsFmSTS1 from F. multiflora that was heterologously expressed in transgenic Arabidopsis and resulted in the accumulation of trans-piceid, which was originally not synthesized in Arabidopsis species.
\end{abstract}

Additional key words: CaMV promoter, HPLC detection, Northern blot, Southern blot.

Fallopia multiflora (Thunb.) Haraldson is one of the most important traditional Chinese medicinal plants (Committee 2010, Saier et al. 2018). Pharmacokinetic studies confirmed that stilbenes are the main characteristic components of F. multiflora. Twenty-one stilbene compounds have been separated from $F$. multiflora, including transresveratrol and a variety of 2,3, 5, 4'-tetrahydroxystilbene glucosides (Lin et al. 2015, Xia et al. 2017). Therein, $2,3,5,4$ '-tetrahydroxystilbene-2- $O$ - $\beta$-D-glucopyranoside (THSG, the structure is shown in Fig. 1) has a similar structure with trans-resveratrol and is considered as the most important active component in F. multiflora (Xia et al. 2017). In Chinese medicine, THSG is often used as a chemical marker for the quality control of $F$. multiflora (Zhao et al. 2014).

Plant stilbenes are derived from phenylalanine, and

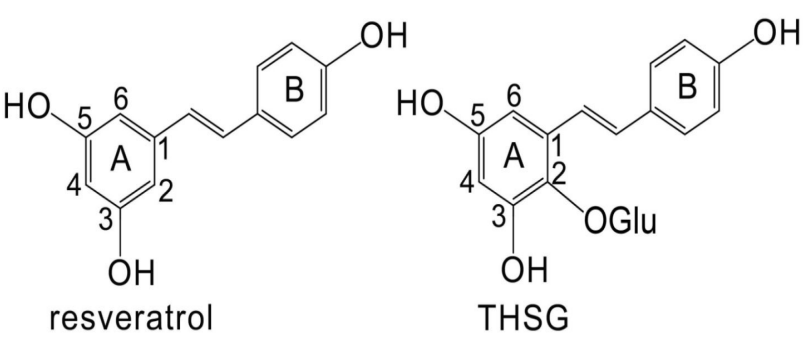

Fig. 1. The stucture of resveratrol and 2,3,5,4'-tetrahydroxy stilbene-2-O- $\beta$-D-glucoside (THSG).

the last step of synthesis is catalysed by stilbene synthase (STS) (Chong et al. 2009) which belongs to type III polyketide synthases (Delaunois et al. 2007, Vannozzi

Submitted 10 June 2019, last revision 17 November 2019, accepted 5 December 2019.

Abbreviations: CaMV - cauliflower mosaic virus; FmSTS1 - Fallopia multiflora stilbene synthase 1; HPLC - high performance liquid chromatography; STS - stilbene synthase.

Acknowledgments: This work was funded by grants from the National Natural Science Foundation of China (CN) (31600236) and the Guangzhou Science Technology and Innovation Commission (201707010459).

* Corresponding author; e-mails: zhaoshujinscut@126.com and scnuchenlei@126.com 

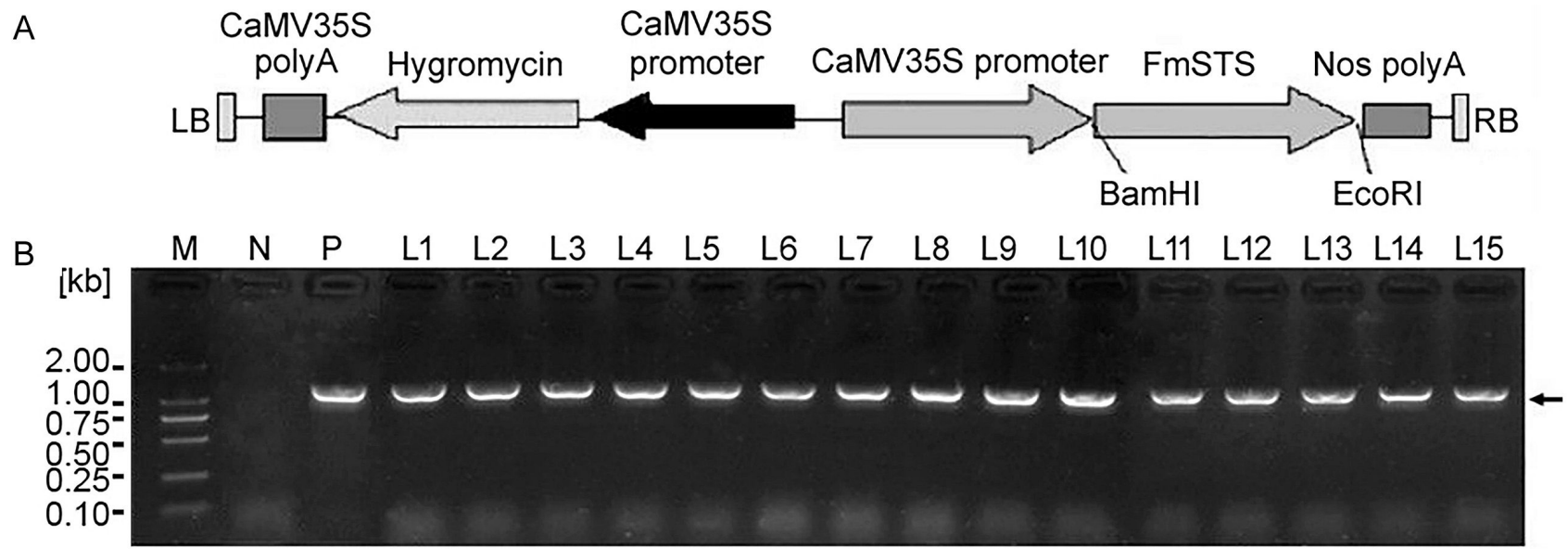

Fig. 2. Identification of transgenic Arabidopsis overexpressing Fallopia multiflora stilbene synthase 1 (FmSTS1). A - A schematic representation of the construct used to overexpress FmSTS1 in Arabidopsis. B - PCR analysis to confirm the presence of the transgene. $\mathrm{N}$ - wild type was used as a negative control; P - plasmid was used as a positive control; L1 to L15 - transgenic Arabidopsis lines of T1 progenies.

et al. 2012). The STS genes have been isolated from many plant species, such as peanut, grapevine, sorghum, and pine (Lu et al. 2016). F. multiflora is a rich source of stilbenes and have several STS genes. Our team has performed a series of studies to elucidate the biosynthesis pathway of THSG in F. multiflora and identified several presumed STS genes through digital gene expression profiling analyses (Zhao et al. 2014).

In our previous study, a presumed STS gene FmSTS1 was isolated from F. multiflora (Sheng et al. 2010). To further confirm its function on the stilbene biosynthesis in F. multiflora, we inserted this gene into Arabidopsis thaliana L. genome for heterologous expression. As a result, a new stilbene compound, which was identified as trans-piceid, accumulated in the transgenic Arabidopsis.

The cDNA of FmSTS1 (NCBI accession No. GQ411431) was PCR amplified with a gene-specific forward primer 5'-AAAGGATCCATGGCAGCTTCAGC-3' and a reverse primer 5'-AAAGAATTCTTAAAAGATGGGCACACTTC-3'. The PCR-amplified FmSTS1 was inserted into a pCAM-1 vector under the cauliflower mosaic virus (CaMV) $35 \mathrm{~S}$ promoter (Fig. 2). The recombinant 35S:FmSTS1 plasmid was transferred into Agrobacterium tumefaciens strain EHA105 by electroporation and then transformed into Arabidopsis thaliana (ecotype Columbia) by the floral dip method (Clough and Bent 1998). For the selection of transformants, sterilized seeds were germinated on agar plates containing hygromycin $\left(25 \mu \mathrm{g} \mathrm{cm} \mathrm{cm}^{-3}\right)$. After 2 weeks of selection, hygromycin-resistant plantlets were transplanted to soil. Transformed plants were identified by PCR and Southern blot analysis. Northern blot analysis was performed to investigate FmSTS1 expression.

Approximately $10 \mu \mathrm{g}$ of genomic DNAs were extracted from the leaves of wild-type and transgenic Arabidopsis. After complete digestion with EcoRI overnight, the samples were isolated by electrophoresis and transferred onto Hybond- $N^{+}$nylon membrane (Amersham Pharmacia, Buckinghamshire, UK) (Zhou et al. 2010). The FmSTS1 cDNA was labeled and used for filter hybridization with a
DIG-High Prime kit (Roche, Mannheim, Germany).

For Northern blot, total RNA was separated from the leaves of wild-type and transgenic Arabidopsis plants. Approximately $20 \mu \mathrm{g}$ of RNA was size fractioned by electrophoresis and transferred to a Hybond $-N^{+}$nylon membrane. Probe preparation and detection were performed as described above for Southern blot analysis.

Transgenic lines with strong FmSTS1 expression were screened until T3 progenies and analyzed for the presence of stilbene-related metabolites. Analysis was performed using the method previously carried out by our team (Liu et al. 2011). Triplicate samples ( $0.5 \mathrm{~g}$ each) were collected from the leaves of 4-week-old T3 plants and ground to fine powder in liquid nitrogen. Ground tissues were extracted with $5 \mathrm{~cm}^{3}$ of $80 \%(\mathrm{v} / \mathrm{v})$ methanol, and supernatants were collected after centrifugation. The methanol fraction was evaporated to dryness under nitrogen. The residue was immediately redissolved in $0.5 \mathrm{~cm}^{3}$ of $80 \%$ methanol. After being filtered through a $0.22 \mu \mathrm{m}$ film, $0.01 \mathrm{~cm}^{3}$ of filtrate was analyzed on an HP 1100 series high performance liquid chromatography (HPLC) system (Agilent Technologies, Palo Alto, CA, USA) by using a Nucleosil C18 column (particle size $5 \mu \mathrm{m}, 4.6 \times 250 \mathrm{~mm}$, Macherey-Nagel, Düren, Germany) and water-acetonitrile as eluent (acetonitrile/water, 25/75, v/v) at flow rate $0.2 \mathrm{~cm}^{3} \mathrm{~min}^{-1}$. A $306 \mathrm{~nm}$ detection wavelength was used.

Liquid chromatography effluent was then introduced into a turbo ion-spray source on a $Q / S T A R-X L$ quadrupole/ time-of-flight (TOF) hybrid mass spectrometer (Applied Biosystems, Foster City, CA, USA). Negative ESI mass spectra were acquired over the range of $100-400 \mathrm{~m} / \mathrm{z}$. The electrospray voltage was set at $-4.5 \mathrm{kV}$, and the source temperature was maintained at $350{ }^{\circ} \mathrm{C}$.

The cDNA of FmSTS1 was expressed in Arabidopsis under the control of a CaMV 35S promoter (Fig. 2A). Fifteen independent hygromycin-resistant transgenic Arabidopsis lines named L1 - L15 were regenerated, and the presence of FmSTS1 was confirmed by PCR (Fig. 2B). Putative transgenic and control plants were selected for 

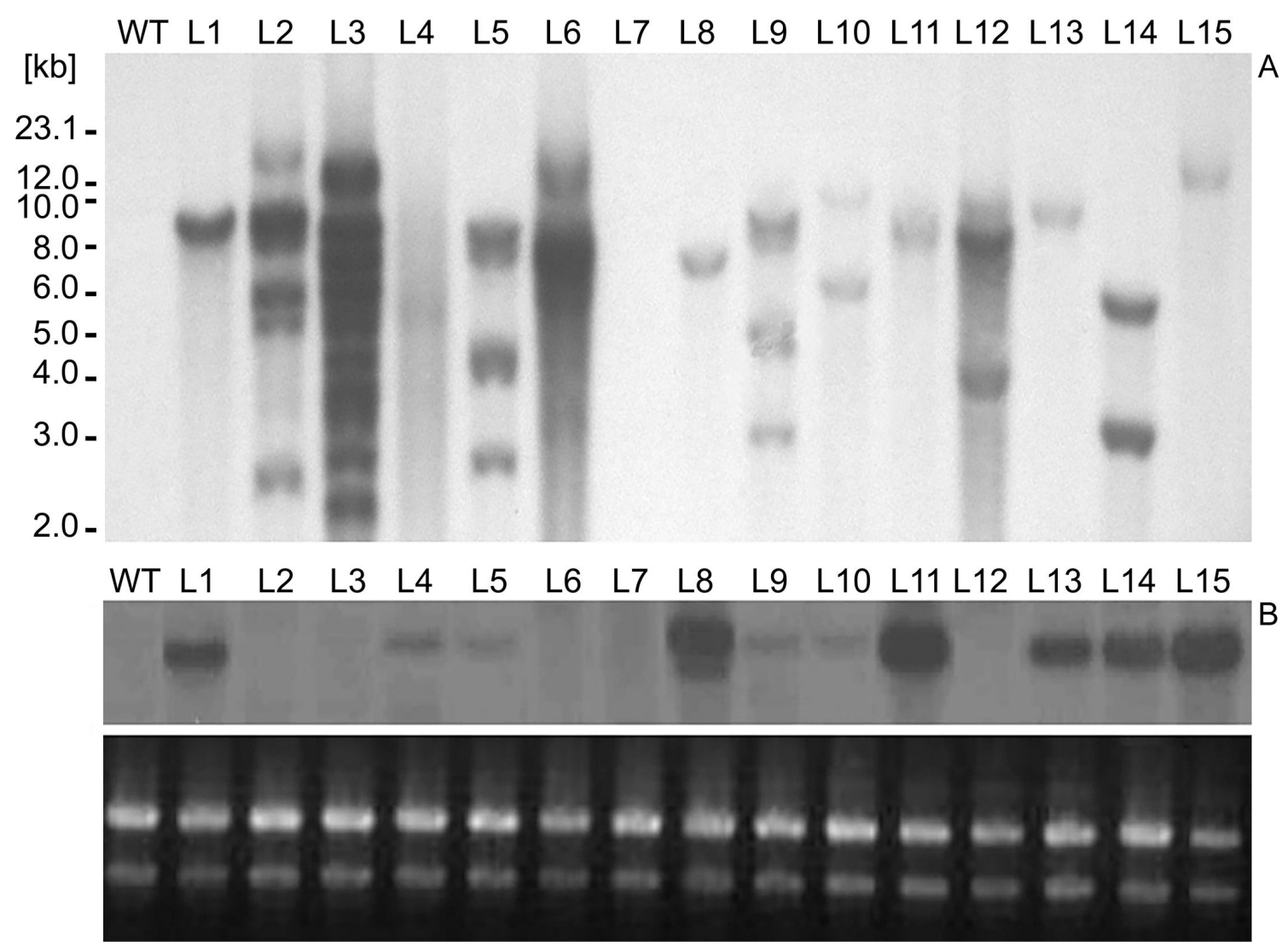

Fig. 3. Southern and Northern blot analyses. $A$ - Southern blot analysis of genomic DNAs digested with EcoRI and hybridized with Fallopia multiflora stilbene synthase 1 (FmSTS1) cDNA. L1, L4, L8, L10, L11, L13, L14, and L15 represent single or low copy integration in the transgenic Arabidopsis lines of T1 progenies. B - Northern blot analysis of transgenic Arabidopsis using PCR amplified FmSTS1 as a probe. L1, L8, L11, L13, L14, and L15 represent relatively high expression of FmSTS1. rRNA was used as a loading control. WT - wild-type.

further studies.

Genomic DNAs from representative transformants and control plants were digested with EcoRI. Southern blot results indicated that besides L7, FmSTS1 was inserted in all other transgenic lines. L2, L3, L5, L6, L9, and L12 contained multiple copies of the transgene. L1, L4, L8, L11, L13, and L15 contained a single copy (Fig. 3A). Northern blot analysis demonstrated no hybridization signal in the untransformed control (Fig. $3 B$ ). The singlecopy- and low-copy-carrying transgenic lines (L1, L8, L11, L13, L14, and L15) showed a relatively high expression of FmSTS1. However, the high-copy transgenic lines (L2, L3, L5, L6, L9, L10, and L12) showed relatively low or absent expression. A comparison of data from Southern blot analysis and those obtained by Northern blot analysis indicates that FmSTS1 expression negatively correlated with transgene copy number. A high copy number of the transgene together with the reduced expression of gene product suggests the occurrence of homology-dependent gene silencing in these lines (Tang et al. 2007, Liu et al. 2011).

The six transgenic lines (L1, L8, L11, L13, L14, and L15) with high expression of FmSTS1 were selected for chemical analysis. HPLC and HPLC-ESI-MS were performed to analyze the stilbene metabolites in the leaves of T3 transgenic lines. Compared with the extracts of the wild type plants, transgenic plants contained an additional peak (Fig. 1 Suppl.). The peak was speculated as transpiceid according to the retention time of $7.5 \mathrm{~min}$, which was the same as the trans-piceid standard (Fig. 1 Suppl.). The peak was then analyzed by HPLC-ESI-MS (Fig. 2 Suppl.). The spectrum of the $[\mathrm{M}-\mathrm{H}]-$ ion $(\mathrm{m} / \mathrm{z}$ 389) with a prominent $\left[\mathrm{M}-\mathrm{H}-\mathrm{C}_{6} \mathrm{H}_{10} \mathrm{O}_{5}\right]$ - product ion at $\mathrm{m} / \mathrm{z} 227$ confirmed the new synthesized compound in transgenic plants as trans-piceid (Liu et al. 2011).

In this study, the products of FmSTS1 found in the leaf tissue of T3 progenies of transgenic Arabidopsis were identified as trans-resveratrol glucosides (trans-piceid) by HPLC and LC-ESI-MS analyses. This result is consistent with the reports on purple sweet potato (Pan et al. 2012), Nicotiana benthamiana (Condori et al. 2009), white poplar (Giorcelli et al. 2004), kiwi (Kobayashi et al. 2000), and transgenic apple fruit (Rühmann et al. 2006). Resveratrol glycosylation is commonly found in plants and has been detected in many STS transgenic lines. This modification is an effective way for resveratrol storage as it can protect resveratrol from oxidation and degradation (Giovinazzo et al. 2012; Lu et al. 2016).

For the six selected transgenic lines, trans-piceid content estimated by HPLC ranged from 46.2 to 65.3 
Table 1. Trans-piceid content [ $\mu \mathrm{g}$ g-1(f.m.)] estimated by HPLC analysis from leaves of $\mathrm{T} 3$ plants. The values represent means \pm SDs. Each experiment was repeated 3 times and included 15 individual plants.

\begin{tabular}{ll}
\hline Line & Trans-piceid content \\
\hline Wild-type & 0.00 \\
L1 & $62.24 \pm 1.74$ \\
L8 & $53.47 \pm 2.06$ \\
L11 & $65.32 \pm 2.18$ \\
L13 & $46.24 \pm 1.46$ \\
L14 & $58.64 \pm 1.87$ \\
L15 & $56.38 \pm 1.83$ \\
\hline
\end{tabular}

$\mu \mathrm{g} \mathrm{g}^{-1}$ (f.m.) (Table 1). Compared with previously published data, this study obtained stilbene content that was lower than that in transgenic Arabidopsis overexpressing a sorghum STS gene (up to $580 \mu \mathrm{g} \mathrm{g} \mathrm{g}^{-1}$ (f.m.) (Hipskind and Paiva 2000). The value is also lower than that in transgenic white poplar with a content of $309-615 \mu \mathrm{g} \mathrm{g} \mathrm{g}^{-1}$ (f.m.) (Giorcelli et al. 2004). However, in some other reports, the anticipated resveratrol or its derivatives were not detected. For example, in transgenic wheat (Serazetdinova et al. 2005), the expression of STS caused the accumulation of unknown stilbene derivatives. In transgenic strawberry (Hanhineva et al. 2009), STS gene transfer caused alterations in the phenylpropanoid metabolism. In F. multiflora, stilbene content is very different because of distinct growth areas. In Chinese pharmacopoeia, THSG content should not be less than $1 \%$ in the dried root of F. multiflora. We determined the THSG content in our experimental sample of $F$. multiflora as high as $14.62 \mathrm{mg}$ $\mathrm{g}^{-1}$ (f.m.) (Zhu et al. 2012). The content of THSG in $F$. multiflora is far higher than that in all of the STS transgenic plants. However, STS transgenic studies developed an effective way to improve nutritional quality or enhance the pathogenic resistance of plants.

Stilbene production in transgenic plants may be related to the promoters selected to drive the transgene, STS genes from different origins, and transgenic plant species. To date, genome sequencing has revealed a large array of STS genes present in plants of different origins. However, only a few are used for the metabolic engineering of plants. Therein, Stsy and Vst 1 from grape are the most commonly used genes (Delaunois et al. 2009). Other STS-encoding genes, such as AhRS gene from peanut (Hipskind et al. 2000), an STS gene from sorghum (Yu et al. 2006), an STS gene from Polygonum cuspidatum (Liu et al. 2011), and an STS gene from Parthenocissus henryana (Jeandet et al. 2018), have also been used. This study demonstrated that in transgenic Arabidopsis, the overexpression of the FmSTS1 gene from F. multiflora leads to the accumulation of trans-piceid. Thus, FmSTS1 provides a new resource for STS metabolic engineering.

THSG is the main component distributed in F. multiflora. Our team has performed considerable research aimed at characterizing the THSG biosynthesis mechanism (Xia et al. 2017). We previously showed that the expression pattern of FmSTS1 analyzed by Northern blot corresponded well with the THSG distribution in different tissues of $F$. multiflora, implying that FmSTS1 may play a crucial role in THSG biosynthesis (Sheng et al. 2010). In the current study, the anticipated THSG was not detected in the transgenic Arabidopsis. However, trans-piceid, 2-hydroxyl glycoside less than THSG, was detected, which may be attributed to the lack of specific hydroxylase in Arabidopsis. Given the structural similarity of piceid and THSG (Fig. 1), THSG may be synthesized by the hydroxylation of trans-resveratrol to form the corresponding tetrahydroxystilbene. This hypothesis was recently confirmed by our intro studies using stable isotope labeling and biocatalytic methods (Xia et al. 2017). Considering the results of previous and present study, we presumed that if FmSTS1 was cotransformed with a hydroxylase from $F$. multiflora, THSG may probably be produced in transgenic Arabidopsis. FmSTS will be transformed into F. multiflora cell line or hair root in future studies to confirm whether it can improve THSG production.

In conclusion, this study shows that overexpression the STS gene FmSTS1 from F. multiflora in transgenic Arabidopsis leads to the accumulation of stilbenes identified as trans-piceid. To our knowledge, this is the first report that an STS gene from F. multiflora transferred to other plants leads to stilbene accumulation. Considering the relevance of the FmSTS1 expression pattern with the distribution of THSG reported in our previous research (Sheng et al. 2010), FmSTS1 may play a crucial role in stilbenes biosynthesis in F. multiflora and may directly affect THSG biosynthesis. Further research will focus on its transformation into F. multiflora cell line or hair root to confirm whether it can improve THSG production.

\section{References}

Chong, J., Poutaraud, A., Hugueney, P.: Metabolism and roles of stilbenes in plants. - Plant Sci 177: 143-155, 2009.

Clough, S.J., Bent, A.F.: Floral dip: a simplified method for Agrobacterium-mediated transformation of Arabidopsis thaliana. - Plant J. 16: 735-743, 1998.

Committee, S.P.: Pharmacopoeia of the People's Republic of China. - People's Medical Publishing House, Beijing 2010.

Condori, J., Medrano, G., Sivakumar, G., Nair, V., Cramer, C., Medina-Bolivar, F.: Functional characterization of a stilbene synthase gene using a transient expression system in planta. Plant Cell Rep. 28: 589-599, 2009.

Delaunois, B., Cordelier, S., Conreux, A., Clément, C., Jeandet, P.: Molecular engineering of resveratrol in plants. - Plant Biotechnol. J. 7: 2-12, 2009.

Giorcelli, A., Sparvoli, F., Mattivi, F., Tava, A., Balestrazzi, A., Vrhovsek, U., Calligari, P., Bollini, R., Confalonieri, M.: Expression of the stilbene synthase (StSy) gene from grapevine in transgenic white poplar results in high accumulation of the antioxidant resveratrol glucosides. - Transgenic Res. 13: 203 214, 2004.

Giovinazzo, G., Ingrosso, I., Paradiso, A., Gara, L. D., Santino, A.: Resveratrol biosynthesis: plant metabolic engineering for nutritional improvement of food. - Plant Foods Human Nutr. 67: 191-199, 2012.

Hanhineva, K., Kokko, H., Siljanen, H., Rogachev, I., Aharoni, 
A., Karenlampi, S. O.: Stilbene synthase gene transfer caused alterations in the phenylpropanoid metabolism of transgenic strawberry (Fragaria $\times$ ananassa). - J. exp. Bot. 60: 20932106, 2009.

Hipskind, J.D., Paiva, N.L.: Constitutive accumulation of a resveratrol-glucoside in transgenic alfalfa increases resistance to Phoma medicaginis. - Mol. Plant Microbe Interact. 13: 551-562, 2000.

Jeandet, P., Sobarzo-Sánchez, E., Clément, C., Nabavi, S.F., Habtemariam, S., Nabavi, S.M., Cordelier, S.: Engineering stilbene metabolic pathways in microbial cells. - Biotechnol. Adv. 36: 2264-2283, 2018.

Kobayashi, S., Ding, C. K., Nakamura, Y., Nakajima, I., Matsumoto, R.: Kiwifruits (Actinidia deliciosa) transformed with a Vitis stilbene synthase gene produce piceid (resveratrolglucoside). - Plant Cell Rep. 19: 904-910, 2000.

Lin, L., Ni, B., Lin, H., Zhang, M., Li, X., Yin, X., Qu, C., Ni, J.: Traditional usages, botany, phytochemistry, pharmacology and toxicology of Polygonum multiflorum Thunb.: a review. J. Ethnopharmacol. 159: 158-183, 2015.

Liu, Z., Zhuang, C., Sheng, S., Shao, L., Zhao, W., Zhao, S.: Overexpression of a resveratrol synthase gene $(P C R S)$ from Polygonum cuspidatum in transgenic Arabidopsis causes the accumulation of trans-piceid with antifungal activity. - Plant Cell Rep. 30: 2027-2036, 2011.

Lu, Y., Shao, D., Shi, J., Huang, Q., Yang, H., Jin, M..: Strategies for enhancing resveratrol production and the expression of pathway enzymes. - Appl. Microbiol. Biotechnol. 100: 74077421, 2016.

Pan, L., Yu, S., Chen, C., Li, H., Wu, Y., Li, H.: Cloning a peanut resveratrol synthase gene and its expression in purple sweet potato. - Plant Cell Rep. 31: 121-131, 2012.

Rühmann, S., Treutter, D., Fritsche, S., Briviba, K., Szankowski, I.: Piceid (resveratrol glucoside) synthesis in stilbene synthase transgenic apple fruit. - J. Agr. Food Chem. 54: 4633-4640, 2006.

Saier, C., Büchter, C., Koch, K., Wätjen, W.: Polygonum multiflorum extract exerts antioxidative effects and increases life span and stress resistance in the model organism Caenorhabditis elegans via DAF-16 and SIR-2.1. - Plants 7:
60-70, 2018.

Serazetdinova, L., Oldach, K.H., Lörz, H.: Expression of transgenic stilbene synthases in wheat causes the accumulation of unknown stilbene derivatives with antifungal activity. - J. Plant Physiol. 162: 985-1002, 2005.

Sheng, S., Liu, Z., Zhao, W., Shao, L., Zhao, S.: Molecular analysis of a type III polyketide synthase gene in Fallopia multiflora. - Biologia 65: 939-946, 2010.

Tang, W., Newton, R. J., Weidner, D.A.: Genetic transformation and gene silencing mediated by multiple copies of a transgene in eastern white pine. - J. exp. Bot. 58: 545-554, 2007.

Vannozzi, A., Dry, I. B., Fasoli, M., Zenoni, S., Lucchin, M.: Genome-wide analysis of the grapevine stilbene synthase multigenic family: genomic organization and expression profiles upon biotic and abiotic stresses. - BMC Plant Biol. 12: 1-22, 2012.

Xia, W., Rui, W., Zhao, W., Sheng, S., Lei, L., Feng, Y., Zhao, S.: Stable isotope labeling and 2,3,5,4'-tetrahydroxystilbene-2-O- $\beta$-D-glucopyranoside biosynthetic pathway characterization in Fallopia multiflora. - Planta 247: 613-623, 2017.

Yu, C., Lam, C., Springob, K., Schmidt, J., Chu, I., Lo, C.: Constitutive accumulation of cis-piceid in transgenic Arabidopsis overexpressing a sorghum stilbene synthase gene. - Plant Cell Physiol. 47: 1017-1021, 2006.

Zhao, W., Xia, W., Li, J., Sheng, S., Lei, L., Zhao, S.: Transcriptome profiling and digital gene expression analysis of Fallopia multiflora to discover putative genes involved in the biosynthesis of $2,3,5,4$ '-tetrahydroxy stilbene-2-O- $\beta$-Dglucoside. - Gene 547: 126-135, 2014.

Zhou, W., Huang, C., Gong, Y.: Molecular cloning and expression analysis of an $A N S$ gene encoding anthocyanidin synthase from purple-fleshed sweet potato [Ipomoea batatas (L.) Lam]. - Plant mol. Biol. Rep. 28: 112-121, 2010.

Zhu, K., Sheng, S., Zhao, W., Lu, D., Xia, W., Zhao, S.: [The content of stilbene glycoside in different parts of Polygonum multiflorum Thunb and the stilbene synthase FM-STS spatial and temporal expression. ] - Progr. modern Biomed. 12: 58195822, 2012. [In Chinese] 